\title{
Towards the Saturation Throughput Disparity of Flows in Directional CSMA/CA Networks: An Analytical Model
}

\author{
Jianrui Fan ${ }^{1}$, Xinru Zhao ${ }^{1}$, Wencan Wang ${ }^{2}$, Shengsuo Cai ${ }^{1 *}$, and Lijuan Zhang ${ }^{1}$ \\ ${ }^{1}$ College of Electronic and Information Engineering, \\ Nanjing University of Aeronautics and Astronautics, Nanjing 210016, China \\ [e-mail: 1120024468@qq.com, zhaoxinru@ nuaa.edu.cn, caishengsuo@ nuaa.edu.cn, lijuan.zhang@nuaa.edu.cn] \\ ${ }^{2}$ Beijing Institute of Astronautical System Engineering, Beijing 100076, China \\ [e-mail: epvolcano@163.com] \\ *Corresponding author: Shengsuo Cai
}

Received September 19, 2020; revised November 8, 2020; accepted December 13, 2020; published April 30, 2021

\begin{abstract}
Using directional antennas in wireless Ad hoc networks has many superiorities, including reducing interference, extending transmission range, and increasing space division multiplexing. However, directional transmission introduces two problems: deafness and directional hidden terminals problems. We observe that these problems result in saturation throughput disparity among the competing flows in directional CSMA/CA based Ad hoc networks and bring challenges for modeling the saturation throughput of the flows. In this article, we concentrate on how to model and analyze the saturation throughput disparity of different flows in directional CSMA/CA based Ad hoc networks. We first divide the collisions occurring in the transmission process into directional instantaneous collisions and directional persistent collisions. Then we propose a four-dimensional Markov chain to analyze the transmission state for a specific node. Our model has three different kinds of processes, namely back-off process, transmission process and freezing process. Each process contains a certain amount of continuous time slots which is defined as the basic time unit of the directional CSMA/CA protocols and the time length of each slot is fixed. We characterize the collision probabilities of the node by the one-step transition probability matrix in our Markov chain model. Accordingly, we can finally deduce the saturation throughput for each directional data stream and evaluate saturation throughput disparity for a given network topology. Finally, we verify the accuracy of our model by comparing the deviation of analytical results and simulation results.
\end{abstract}

Keywords: Ad Hoc Networks, Directional CSMA/CA Protocol, Saturation Throughput Disparity, Markov Chain Model

This work is partly supported by the National Natural Science Foundation of China (61902182), the Natural Science Foundation of Jiangsu Province of China (BK20190409), China Postdoctoral Science Foundation (2019TQ0153), Graduate Innovation Base (Lab) Open Foundation of Nanjing University of Aeronautics and Astronautics (kfjj20190413). 


\section{Introduction}

In recent years, motivated by the rapid developments of new applications in wireless Ad hoc networks, including traffic control [1], border surveillance [2], disaster relief [3], precision agriculture [4], etc., there has emerged a great demand for improving the network throughput [5]. The broadcast nature of traditional omni-directional antennas limits the upper bound of the network throughput due to overmuch interferences among multi-users. Using directional antennas can effectively make up this drawback in wireless Ad hoc networks [6]. When comparing with omni-directional antennas, directional antennas are able to provide higher spatial reuse by allowing more concurrent data transmissions among multiple node pairs. The directional antennas can also enhance the anti-interference ability of wireless devices that are affected by jamming phenomena [7].

In order to make full use of the advantages of directional antennas, a lot of work has been done on how to design the medium access control (MAC) protocols using directional randomaccess scheme [8-15]. Choudhury et al. [8] proposed a basic directional MAC protocol (basic DMAC) to exploit the characteristics of directional antennas. Shihab et al. [9] proposed a directional-to-directional (DtD) MAC protocol and analyzed the saturation throughput for DtD MAC through a theoretical model. To support more concurrent transmissions, the authors in [10] proposed a multi-channel directional MAC protocol called MMAC-DA combining the advantages of multi-channel and directional antennas. In [11], a random-access directional MAC protocol for high-density UAV networks was proposed, which uses a tone-based energy detection scheme to alleviate the impact of deafness problem. Considering both frequent topology changes and time-varying flight environments, Zheng et al. [12] proposed a positionprediction-based directional MAC protocol (PPMAC) for flying Ad hoc networks (FANETs), which aims to create smarter and more self-ruling communication for FANETs. The authors in [13] paid attention to the underwater acoustic sensor networks (UASNs) and proposed a dual-channel directional MAC protocol (DADC-MAC) for UASNs. Akntar [14] proposed the Directional MAC protocol for Basic Stations (DMBS) with the goal of fully leveraging spatial reusability and limiting deafness and hidden terminal problems with minimal overhead, and without using any complicated hardware for localization. And Quynh [15] developed a novel directional medium access control scheme with an extreme power saving mechanism is developed for traffic indication map stations in the IEEE 802.11ah networks.

The above random-access directional MAC protocols have one thing in common: they are all based on the carrier sense multiple access with collision avoidance (CSMA/CA) scheme. In CSMA/CA based Ad hoc networks, the transmitting node should sense the channel state before its transmission. If a node hears a transmission process of another node, it will defer its transmission, so the collision is avoided. However, when using directional antennas, these CSMA/CA based MAC protocols are suffered from the directional antennas-related problems, such as deafness problem and directional hidden terminal problem [16]. Due to these directional antennas-related problems, some collisions that can be avoided through CSMA/CA no longer avoidable. From the network view, these problems make the network environment faced by each node different, which subsequently leads to the saturation throughput disparity of flows in directional CSMA/CA based Ad hoc networks. The saturation throughput disparity may reduce the quality of service (QOS) experienced by flows, i.e., some flows can achieve very high throughput while the rest of the flows achieve rather low throughput. In some extreme situations, it will cause severe unfairness and even flow starvation [17].

Aiming at the unfairness phenomena caused by the saturation throughput disparity in directional CSMA/CA based Ad hoc networks, researchers have made some attempts to 
alleviate it $[9,13,18]$. The authors in [9] adjusted the request-to-send/clear to send (RTS-CTS) mode by sending RTS frames in each beam direction to alleviate the deafness problem. In [13], the communication channel is segmented into two sub-channels, one sub-channel for data transmission and the other for busy prompt message transmission to broadcast the on-going communications. In [18], the authors suggested that the deafness problem can be alleviated by location estimation of neighbor nodes. However, these methods are all lack of theoretical foundations and cannot quantify the impact of the directional antennas-related problems on the saturation throughput disparity of flows. Modeling this common behavior of directional CSMA/CA based Ad hoc networks is of great significance in the development of corresponding strategies to deal with the unfairness problems caused by saturation throughput disparity as well as in MAC protocol design.

In this article, we concentrate on modeling and analyzing the saturation throughput disparity of flows in CSMA/CA based ad hoc networks. We choose a typical directional CSMA/CA based MAC protocol, the basic DMAC [8], as the case of our modeling scheme. In general, the main contributions of our work include: (i) We creatively analyze the limitations of traditional Markov chain model based on variable-length slot in modeling the directional MAC protocol, while there are no one else had done, to the best of our knowledge; (ii) We propose two kinds of collisions: instantaneous collision probability and persistent collision probability to count the impact of the directional antennas-related problems on the saturation throughput disparity, and the difference of these two kind of collisions is also the most fundamental reason of saturation throughput disparity; (iii) We establish a four-dimensional Markov chain model based on fixed-length slot. This model can provide a deeper understanding of the flow saturation throughput disparity in directional CSMA/CA based Ad hoc networks and it has important theoretical guiding significance for the design and improvement of directional network protocols.

The rest of the paper is organized as follows. In section 2, we briefly review the related analytical model on CSMA/CA based MAC protocols. Section 3 explains the limitation of variable-length slot Markov chain in modeling the directional MAC protocol. In section 4, we propose our four-dimensional fixed-length Markov chain model, and the expressions of the collision probability, the transmission probability and the per-flow saturation throughput are derived. Section 5 compares the results of the analytical model with the extensive simulation results. Finally, we conclude this paper in Section 6.

\section{Related Works}

The saturation throughput, which is one of the most important performance indicators of the Ad hoc networks, is defined as the upper limit reached by the network throughput with the offered load increasing. The modeling and analyzing the saturation throughput has always been a hot topic in the field of CSMA/CA based Ad hoc networks. One of the most famous is the Markov chain model proposed by Bianchi for CSMA/CA based Ad hoc networks [19]. The model divides the network timeline into consecutive variable-length slots to analyze the behavior of exponential back-off details. It is not complicated but accurate to calculate the saturation throughput and provides a successful example for the follow-up research.

Based on Bianchi's work, Felemban and Ekici [20] proposed an analytical model for CSMA/CA based Ad hoc networks in the single hop setting under both saturated and unsaturated traffic cases. Dai and Sun [21] proposed a unified analytical model to study the stability of homogeneous buffered networks. In [22], a mathematical model was proposed to compare the throughput and packet delay for directional CSMA/CA based Ad hoc networks, 
which adopts a nonideal antennas model and propagation environment. The authors in [23] proposed a generic analytical framework for modeling the saturation throughput which considers the presence of the deafness problem in wireless networks with directional antennas. Chen et al. [24] developed a general theoretical framework to derive the throughput in mobile Ad hoc networks with directional antennas. The authors in [25] studied the throughput performance of directional wireless networks and use a five-dimensional Markov chain to investigate the effect of various network parameters on the achievable saturation throughput. $\mathrm{Wu}$ et al. [26] focused on the efficiency of beamforming training protocols and used an analytical model for optimizing the system parameters to make the network throughput as high as possible.

The fundamental premise of the above work is that the channel state sensed by each node is the same. Based on this assumption, the condition collision probability of every communication node is identical to each other, too. Therefore, the theoretical model only needs to analyze the saturation throughput of an arbitrary node. And at the same time, the saturated throughput of the overall network with $N$ nodes is calculated as the $N$ times of one node. However, the network environment faced by each node is completely different in directional Ad hoc networks due to the directional antennas-related problems like deafness and directional hidden terminal problems. The previous modeling work based on variable-length slot cannot distinguish the saturation throughput disparity of flows. In the next section, we will analyze the limitation of variable-length slot based modeling scheme and its impact on saturation throughput calculation.

\section{Motivation: Limitation of Variable-Length Slot}

\subsection{The Variable-length Slot}

According to the previous statement, we know that most of the works dealing with saturation throughput analysis are based on Bianchi's model [19]. It a discrete-time backoff scale, and the time following an idle distributed interframe, namely distributed inter-frame space (DIFS) is slotted. We use $\varsigma$ to denote this series of "variable-length slot". If assuming $E[\varsigma]$ to denote the expectation of variable-length slot, the saturated throughput $S$ can be defined to be:

$$
S=\frac{E[\text { payload information transmitted in a slot time }]}{E[\varsigma]} .
$$

As we can see, the key of solving saturation throughput relies on the calculation of the average length of variable-length slot. The mathematical expectation $E[\varsigma]$ of variable-length slot depends on the probability of each state of the channel, i.e., with probability $P_{\text {idle }}$ that the channel is idle, with probability $P_{\text {suc }}$ that the channel contains a successful transmission, and with probability $P_{\text {fail }}$ that this channel experiences a collision. Therefore, $E[\varsigma]$ becomes

$$
E[\varsigma]=P_{\text {idle }} T_{\text {idle }}+P_{\text {suc }} T_{\text {suc }}+P_{\text {fail }} T_{\text {fail }} \text {. }
$$




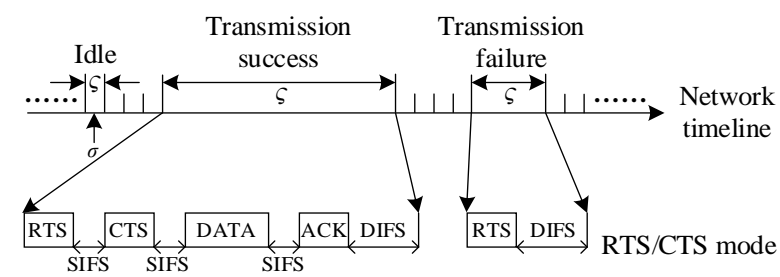

Fig. 1. Distribution of variable-length slot

Here $T_{\text {idle }}$ is the duration of an idle slot, it has the fixed length $\sigma$, which is assumed to be equal to the maximum time-length for one node to detect the transmission of any other node. $T_{\text {suc }}$ represents the duration that a node senses the communication channel has been occupied because of one successful transmitting action, and $T_{\text {fail }}$ represents the duration that the node detects the communication channel has been occupied because of a collision.

Fig. 1 shows the distribution of variable-length slot. As shown in Fig. 1, the value of $T_{\text {suc }}$ and $T_{\text {fail }}$ in RTS/CTS mode are:

$$
\left\{\begin{array}{l}
T_{\text {suc }}^{\mathrm{RTS}}=\mathrm{RTS}+\mathrm{CTS}+\mathrm{DATA}+\mathrm{ACK}+3 \mathrm{SIFS}+\mathrm{DIFS}+4 \delta \\
T_{\text {fail }}^{\mathrm{RTS}}=\mathrm{RTS}+\mathrm{DIFS}+\delta
\end{array}\right.
$$

where RTS, CTS, DATA and ACK represent the transmission delay of the corresponding frame, $\delta$ indicates the propagation delay of a packet, and SIFS (Short Inter-frame Space) represents the minimum inter-frame interval.

\subsection{Limitation Analysis in Directional Ad Hoc Networks}

According to Bianchi's model, all the transitions of nodes between different states are regarded as the same and synchronized. This assumption is reasonable for a single-hop wireless network. Because the propagation delay is much smaller than the minimum-length slot $\sigma$, all nodes can sense the channel state change in the same variable-length slot. In other words, the state of nodes during one variable-length slot will only change at the beginning of it and keeps constant for the rest of the time. As a result, the derivation of the saturation throughput just needs to analyze an arbitrary flow, and each flow shares the same channel bandwidth.

However, in directional CSMA/CA based Ad hoc networks the above assumption is not accurate. For instance, Fig. 2 shows a directional chain topology scenario which consists of two flows: flow $\mathrm{A} \rightarrow \mathrm{B}$, and flow $\mathrm{C} \rightarrow \mathrm{D}$. Although nodes $\mathrm{A}, \mathrm{B}, \mathrm{C}$ and $\mathrm{D}$ are in a single-hop network, node $\mathrm{C}$ cannot detect the node A's RTS frame to node $\mathrm{B}$ and sends its own RTS to node D after the back-off counter reaches zero. Finally, there will be a collision at node D. In fact, because of the directional hidden terminal problem, the variable-length slot of nodes A, B, C and D are no longer synchronized. Nodes B and D sense the channel busy while node C senses the channel idle. This disparity of network environment will eventually lead to the saturation throughput disparity of flows. Therefore, if we still adopt the variable-length slot based modeling scheme, we will not get the right results consistent with the reality.

Table 1 demonstrates the simulation and the theoretical results of the saturation throughput of topology assumed in Fig. 2. The simulation result indicates that the saturation throughput of flow $\mathrm{A} \rightarrow \mathrm{B}$ is much higher than the flow $\mathrm{C} \rightarrow \mathrm{D}$ because of the directional hidden terminal problem introduced by using directional antennas. However, the analytical results based on variable length time slot is that saturation throughput of two flows is the same. Although the total network saturation throughput of simulation and analytical is close, the variable-length 
slot Markov chain model cannot effectively reflect the saturation throughput disparity in a directional wireless network.

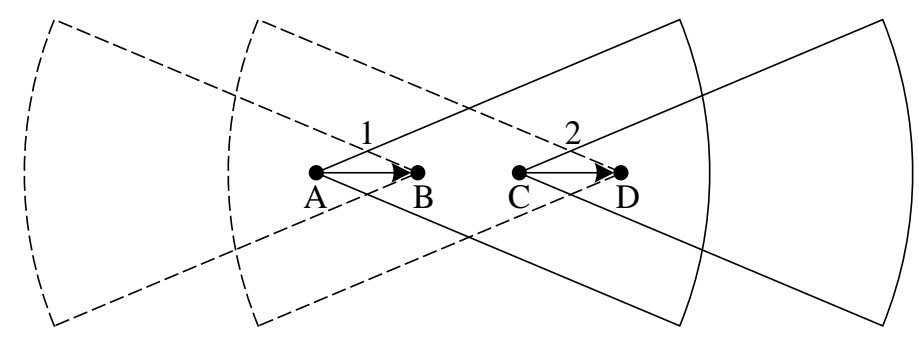

Fig. 2. The assumed chain topology for directional Ad hoc networks

Table 1. Saturation Throughput of Different Flows

\begin{tabular}{|c|c|c|}
\hline Flow & Analytical results based on variable-length slot & Simulation results by NS3 \\
\hline \hline $\mathrm{A} \rightarrow \mathrm{B}$ & $0.407 \mathrm{Mbps}$ & $0.767 \mathrm{Mbps}$ \\
\hline $\mathrm{C} \rightarrow \mathrm{D}$ & $0.407 \mathrm{Mbps}$ & $0.108 \mathrm{Mbps}$ \\
\hline
\end{tabular}

Therefore, we can conclude from the simulation above that when using directional antennas, the variable-length slot is no longer synchronized due to the deafness and directional hidden terminal problems. There is saturation throughput disparity among different flows, and the variable-length slot Markov chain cannot accurately model it.

\section{Analytical Model}

\subsection{The Fixed-length Slot}

As mentioned above, due to the directional antennas-related problems in directional CSMA/CA based Ad hoc networks, the collision occurs not only at the beginning of each slot, but also the rest of the slot. It is a pity that the variable-length slot based modeling scheme cannot distinguish the collision at different moments. To model this behavior in directional CSMA/CA Ad hoc networks, we use the fixed-length slot to divide the collision into instantaneous collision and persistent collision.

The notion of fixed-length slot was first used in [27] to consider the behavior of hidden terminal problem in a multi-hop CSMA/CA based Ad hoc networks. The authors in [24] use "transmission" and "freezing" to represent the communication channel is occupied because of the transmitting state of the node itself and other nodes, respectively. To overcome the limitation that the traditional modeling schemes do not take into account the desynchronization of the freezing process experienced by nodes in a multi-hop CSMA/CA Ad hoc networks, the freezing process is discretized into a succession of fixed-length slots of duration $\sigma$. A basic assumption behind this model is that the duration of the transmission and freezing process is an integer multiple of the fixed-length slot. 


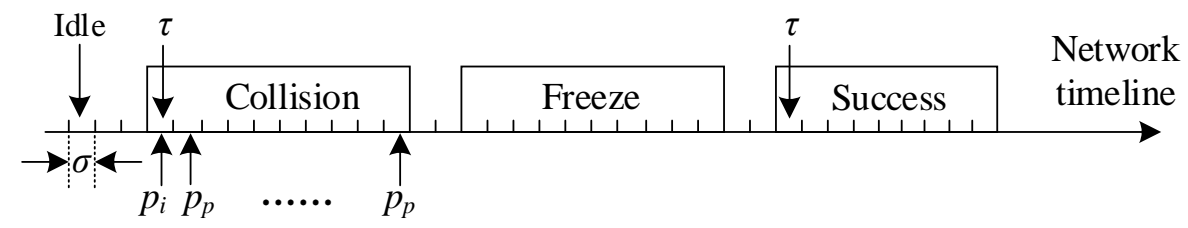

Fig. 3. Distribution of variable length slot

Similarly, we also adopt the notion of fixed-length slot to view the channel state and use the slot time $\sigma$ stated in [19] as the unit time in our model. The difference between our model and [27] is that, as shown in Fig. 3, the whole network timeline is discretized into a succession of fixed-length slots, not only the freezing process but also the transmission process. In addition, all the evaluation metrics are recounted according to the concept of fixed-length time slot. For example, $\tau$ is redefined as the probability that the target node starts transmission in an arbitrary fixed-length slot. The collision probability is divided into instantaneous collision probability $P_{i}$ and persistent collision probability $P_{p} . P_{i}$ represents the possibility of collision in the first time slot of the transmitting process, and $P_{p}$ represents the probability of collision occurs in any slot after the first time slot of the transmitting process. In the following discussions, unless other specified, the term "slot" refers to the fixed-length slot.

\subsection{Collision Probability Analysis}

In basic DMAC, the RTS-CTS handshake mechanism is adopted, and all frames are transmitted with directional antennas. An idle node senses the channel omni-directionally, and once there is a signal, it will adapt its antenna into the directional mode by using Direction-ofArrival (DOA) technique. In other words, there are two types of links in basic DMAC: DtO link (one end node with directional antennas and the other end node with omni-directional antennas) and DtD link (both ends with directional antennas). As we all know, the switch between DtO and DtD mode will cause the hidden terminal problem because of asymmetry in gain and further lead to the failure of CTS transmission. Apart from that, the hidden terminal problem due to unheard RTS/CTS frame or the deafness problem will also lead to the collision of transmission. Next, we will analyze the probability of these collisions at different stages during the entire transmission process in detail.

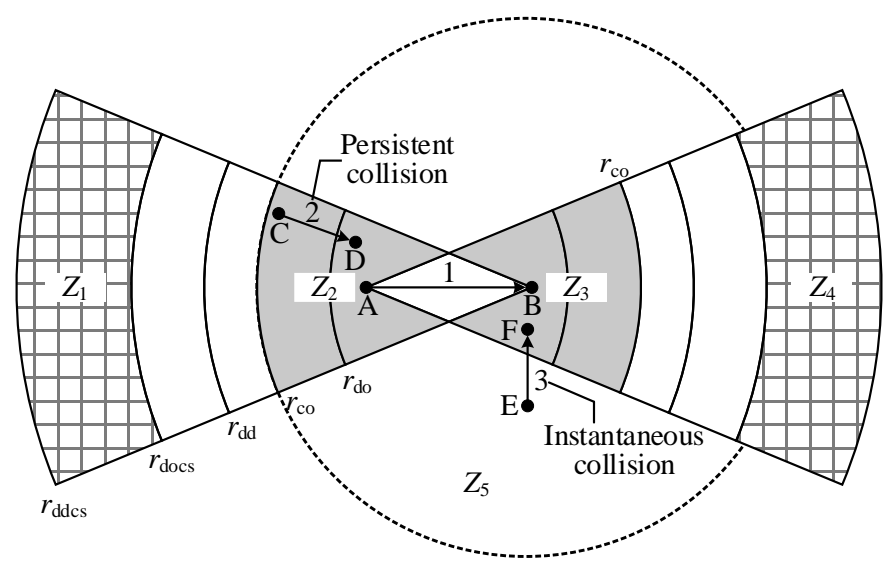

Fig. 4. Collision zone division of flow $\mathrm{A} \rightarrow \mathrm{B}$ 
Table 2. Symbols and Definition

\begin{tabular}{|c|c|}
\hline Symbols & Definition \\
\hline \hline$r_{\mathrm{do}} / r_{\mathrm{dd}}$ & The transmission range in DtO/DtD mode. \\
\hline$r_{\mathrm{docs}} / r_{\mathrm{ddcs}}$ & The carrier sense range in DtO/DtD mode. \\
\hline$r_{\mathrm{co}}$ & The collision range. \\
\hline$Z_{1} / Z_{4}$ & The annular sector area filled with grid lines. \\
\hline$Z_{2} / Z_{3}$ & The irregular area filled with grey. \\
\hline$Z_{5}$ & The circle with B as the center and $r_{\mathrm{co}}$ as the radius. \\
\hline$L_{f}$ & Frame $f(f=\mathrm{RTS} / \mathrm{CTS} / \mathrm{DATA} / \mathrm{ACK})$ lasts for $L$ slots. \\
\hline
\end{tabular}

To illustrate the problems more clearly, we assume a specific scenario with flow $\mathrm{A} \rightarrow \mathrm{B}$ as the tagged flow as shown in Fig. 4. The related symbols and their definition are given in Table 2. In CSMA/CA based Ad hoc networks, if the signals of interfering nodes is in the collision range of the receiving node (just as nodes $\mathrm{C}$ and $\mathrm{E}$ with $\mathrm{B}$ ) arrive at the same time as the sending nodes signal, it will cause a collision. A similar derivation of collision range $r_{\mathrm{co}}$ can be found in [28] (refer to (1) and (2) in [28]). Here, we directly give the results as

$$
r_{\mathrm{co}}=\sqrt[4]{S N R_{\mathrm{th}}} d_{\mathrm{AB}}
$$

where $S N R_{\mathrm{th}}$ is the signal to noise ratio threshold of the receiver, and $d_{\mathrm{AB}}$ represents the communication distance between the sender and receiver. In practice, the $S N R_{\mathrm{th}}$ is usually set to be $10 \mathrm{~dB}$, and thus we get $r_{\mathrm{co}}=1.78 d_{\mathrm{AB}}$. On one hand, we assume that $d_{\mathrm{AB}}$ is greater than $0.56 r_{\mathrm{do}}$, as shown in Fig. 4 . It can be known that the collision range is larger than $r_{\mathrm{do}}$, and the biggest value of $r_{\mathrm{co}}$ is $1.78 r_{\mathrm{do}}$. On the other hand, $r_{\mathrm{dd}}$ is about three times of $r_{\mathrm{do}}$ according to [29]. As a result, the value of $r_{\mathrm{co}}$ is between $r_{\mathrm{do}}$ and $r_{\mathrm{dd}}$.

In Fig. 4, node A senses the current beam idle, and its back-off counter reaches zero. Then it transmits an RTS frame to node B. Assume that the time needed to switch from omnidirectional antennas to directional antennas is less than one slot time. If node $\mathrm{E}$ transmits an RTS frame to node $\mathrm{F}$ at this time, before node B beamforms toward node A's direction, there will be a conflict at node B. Note that this sort of collision only occurs at the first slot during the transmission process, and we call it "instantaneous collision". The area that may cause the instantaneous collision of the receiving node is corresponding named as "instantaneous collision zone". For example, the instantaneous collision zone in Fig. 4 is $Z_{5}$.

The nodes in $Z_{2}$, just like nodes $C$ and $D$, sense the beam idle and cannot hear the RTS frame during the entire RTS transmission process. If node C's finishes its back-off stage during this process, it will transmit an RTS frame to node D. As a result, the RTS frames of node A and $\mathrm{C}$ will cause a collision at node B. Since node $\mathrm{C}$ cannot detect the busy state of channel with directional carrier sense mechanism, it may transmit its RTS frame at any time during the RTS transmission process of node A. In other words, collisions may occur throughout the transmission process. Similarly, we call this kind of collision "persistent collision", and the corresponding collision zone is named as "persistent collision zone".

It can be seen from the above analysis that in directional CSMA/CA based Ad hoc networks, the collisions may occur at any time during the entire transmission process due to the existence of the persistent collision zone. For clarity, we choose flow $\mathrm{A} \rightarrow \mathrm{B}$ as the tagged flow and divide the collisions into two stages.

1) Collisions during RTS-CTS transmission process

The success of RTS transmission depends on two aspects. First, the nodes in $Z_{5}$ cannot be in 
the transmitting process during the first time slot. Second, the nodes in $Z_{2}$ cannot transmit its RTS frame during the remaining $\left(L_{\mathrm{RTS}}-1\right)$ slots. Therefore, we can derive the collision probability of the RTS frame as

$$
p_{\mathrm{RTS}}=1-\prod_{x_{1} \in Z_{\mathcal{S}}, x_{2} \in Z_{2}}\left[1-\tau^{\prime}\left(x_{1}\right)\right]\left[1-\tau_{\mathrm{RTS}}\left(x_{2}\right)\right]^{\mathrm{RTS}_{\mathrm{RT}}-1},
$$

where $\tau^{\prime}$ represents the probability that the current node is at the transmitting process, and $\tau_{\text {RTS }}$ represents the transmission probability of the RTS frame.

After receiving the RTS frame successfully, receiving node B will beamform towards node A's direction and response with a CTS frame. If the nodes in $Z_{2}$ are in the transmitting process during the first time slot or the nodes in $Z_{3}$ transmit the RTS frame at any time during the CTS transmission process, node A will fail to receive the CTS frame. In other words, the collision probability of the CTS frame is

$$
p_{\mathrm{CTS}}=1-\prod_{x_{1} \in Z_{1}, x_{2} \in Z_{3}}\left[1-\tau^{\prime}\left(x_{1}\right)\right]\left[1-\tau_{\mathrm{RTS}}\left(x_{2}\right)\right]^{L_{\mathrm{CTS}}} .
$$

If we denote the probability of collisions during RTS-CTS transmission process as $p_{\mathrm{RC}}$, it can be written as

$$
p_{\mathrm{RC}}=1-\left(1-p_{\mathrm{RTS}}\right)\left(1-p_{\mathrm{CTS}}\right)
$$

2) Collisions during DATA-ACK transmission process

Once nodes A and B have finished the RTS-CTS handshake, there are only persistent collisions. As long as the nodes in $Z_{2}$ do not transmit the RTS frame during the DATA transmission process and the nodes in $Z_{3}$ do not transmit the RTS frame during the ACK transmission process, a successful packet transmission is finished. The collision probability during DATA-ACK transmission process is obtained as

$$
p_{\mathrm{DA}}=1-\prod_{x_{1} \in Z_{2}, x_{2} \in Z_{3}}\left[1-\tau_{\mathrm{RTS}}\left(x_{1}\right)\right]^{L_{\mathrm{DATA}}}\left[1-\tau_{\mathrm{RTS}}\left(x_{2}\right)\right]^{L_{\mathrm{ACK}}} \text {. }
$$

\subsection{Four-dimensional Markov Chain Model}

Based on the notation of fixed-length slot, as shown in Fig. 5, we propose a four-dimensional Markov chain model for basic DMAC. In our model, we consider a discrete and integer time scale, where $t$ and $t+1$ represent two back-off slot times which are adjacent. The state for a given node $n$ at time $t$ can be depicted with a four-dimensional stochastic process $\{i(t), s(t)$, $b(t), k(t)\}$, they respectively represent:

- $i(t)$ is a stochastic process representing the MAC state for a given node at time $t$, where 0 represents the node is in the back-off process, 1 represents the freezing process, 2 represents the RTS-CTS transmission process, and 3 represents the DATA-ACK transmission process.

- $s(t)$ is a stochastic process representing the back-off process in the range from 0 to $m$ for a given node at time $t$.

- $b(t)$ is a stochastic process representing the value of the back-off time counter for one node at time $t$. When $s(t)$ is $j(0 \leq j \leq m), b(t)$ is in the range from 0 to $W_{j-1}$.

- $k(t)$ is a stochastic process representing the number of remaining slots in the transmission or 
freezing process for a given node at time $t$. It should be noted that it is 0 for back-off process.

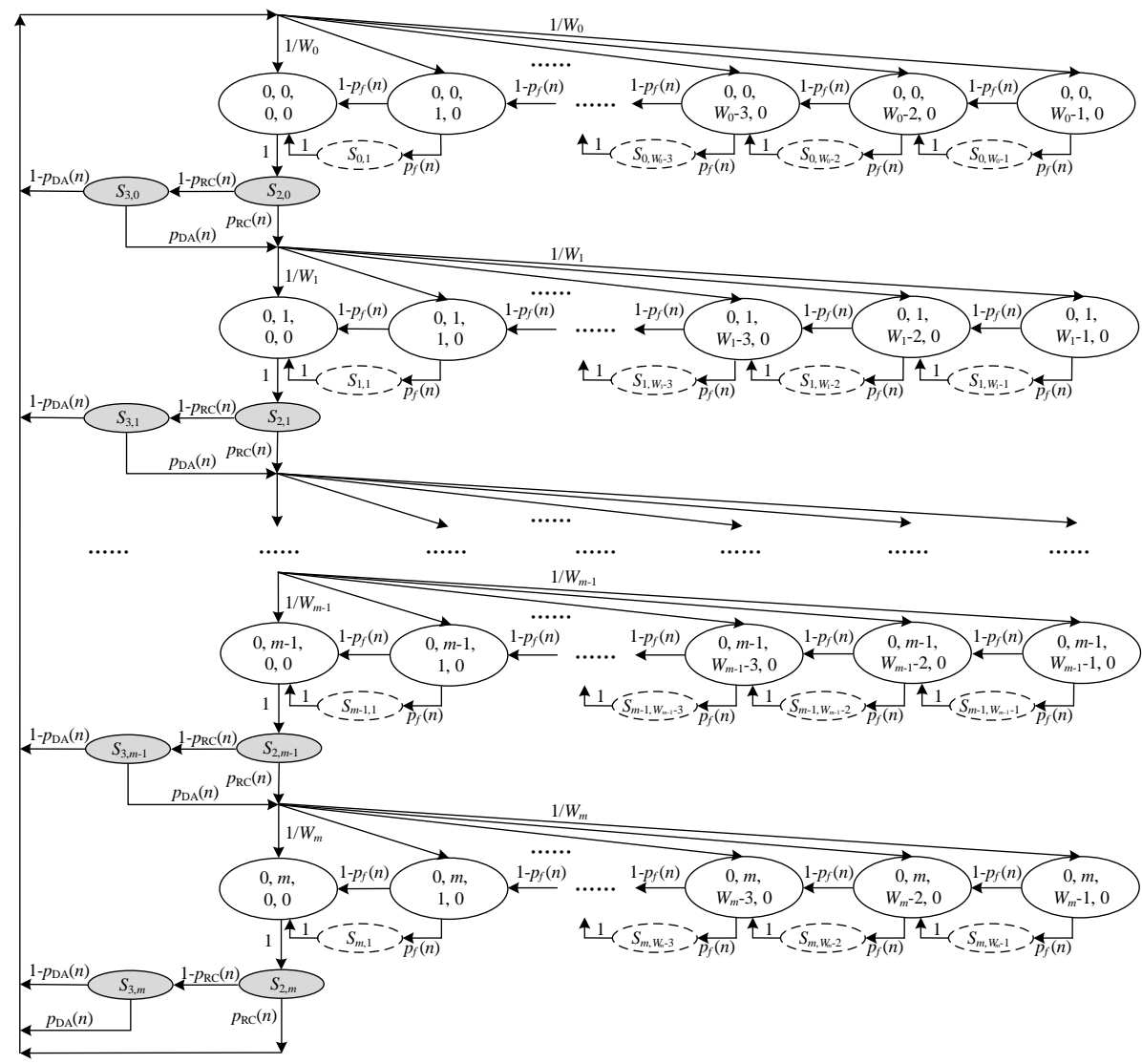

Fig. 5. Four-dimensional Markov chain model

Before transmitting packets, the node should sense the channel state. If the channel remains free for a DIFS time, it will choose a random back-off value and start the back-off process. As demonstrated in Fig. 5, the back-off time counter of current node will decrease by one if the channel is free for one slot time-length. When the channel is occupied during the back-off process, this node will suspend the back-off time counter and start freezing process. The node starts the RTS-CTS transmission process when the back-off time counter reaches zero. And then, this node will enter the DATA-ACK transmitting process with probability $1-p_{\mathrm{RC}}(n)$. Whether the failure of the RTS-CTS transmission or the DATA-ACK transmission, the backoff stage will increase by one and the contention window is doubled. The node will select a new back-off number from the new contention window and attempt to retransmit this packet. If we use $W_{j}$ to represent the contention window size at $j$ th back-off stage, we can get

$$
W_{j}=2^{j} W_{0} \quad 0 \leq j \leq m,
$$

where $W_{0}$ represents the minimum contention window size. When $W_{j}$ reaches its maximum value $W_{\max }$, the node will drop this data packet and try to send the next packet. In the following discussion, we will analyze the above four-dimensional Markov chain mode from three aspects of the back-off process, transmission process and freezing process. 


\subsection{Back-off Process}

Let $p(b \mid a)$ be the possibility of transition for one node from stage " $a$ " to stage " $b$ ". $S_{j, k}$ represents the freezing process when the back-off state is $j$ and the back-off time counter is $k$. $S_{2, j}$ and $S_{3, j}$ represent the RTS-CTS and DATA-ACK transmission process at $j$ th back-off stage respectively. Then the non- null one step transition probabilities are

$$
\begin{cases}p(0, j, k, 0 \mid 0, j, k+1,0)=1-p_{f}(n) & 0 \leq j \leq m, 0 \leq k \leq W_{j}-2 \\ p\left(S_{j, k} \mid 0, j, k, 0\right)=p_{f}(n) & 0 \leq j \leq m, 1 \leq k \leq W_{j}-1 \\ p\left(0, j, k, 0 \mid S_{j, k+1}\right)=1 & 0 \leq j \leq m, 0 \leq k \leq W_{j}-2 \\ p\left(S_{2, j} \mid 0, j, 0,0\right)=1 & 0 \leq j \leq m \\ p\left(0, j+1, k, 0 \mid S_{2, j}\right)=\frac{p_{\mathrm{RC}}(n)}{W_{j+1}} & 0 \leq j \leq m-1,0 \leq k \leq W_{j+1}-1 \\ p\left(S_{3, j} \mid S_{2, j}\right)=1-p_{\mathrm{RC}}(n) & 0 \leq j \leq m \\ p\left(0, j+1, k, 0 \mid S_{3, j}\right)=\frac{p_{\mathrm{DA}}(n)}{W_{j+1}} & 0 \leq j \leq m-1,0 \leq k \leq W_{j+1}-1 \\ p\left(0,0, k, 0 \mid S_{3, j}\right)=\frac{\left[1-p_{\mathrm{DA}}(n)\right]}{W_{0}} & 0 \leq j \leq m, 0 \leq k \leq W_{0}-1 \\ p\left(0,0, k, 0 \mid S_{2, j}\right)=\frac{p_{\mathrm{RC}}(n)}{W_{0}} & 0 \leq k \leq W_{0}-1 \\ p\left(0,0, k, 0 \mid S_{3, j}\right)=1 / W_{0} & 0 \leq k \leq W_{0}-1\end{cases}
$$

where $p_{f}(n)$ represents the freezing probability of node $n, p_{\mathrm{RC}}(n)$ and $p_{\mathrm{DA}}(n)$ represent the RTCCTS and DATA-ACK collision probability of node $n$, respectively. The equations in (10) are explained as follows:

- (10a) (10c) depict the back-off and freezing process of node $n$. The back-off time counter will be decremented by 1 with probability $1-p_{f}(n)$ and be suspended with probability $p_{f}(n)$. After the freezing process finishes, it will continue the back-off process with probability 1.

- (10d) (10f) depict the RTS-CTS transmission process. 10(d) represents node $n$ starts to transmit RTS with probability 1 when the value of back-off time counter reaches zero. 10(e) represents that node $n$ enters DATA-ACK transmission process with probability $1-p_{\mathrm{RC}}(n)$ and 10(f) represents that it enters the back-off process of the next stage with probability $p_{\mathrm{RC}}(n)$.

- $(10 \mathrm{~g})$ and $(10 \mathrm{~h})$ depict the DATA-ACK transmission process. $10(\mathrm{~g})$ represents that the transmission is failed with probability $p_{\mathrm{DA}}(n)$ and node $n$ enters the back-off process of the next stage. 10(h) represents that the transmission is successful with probability $p_{\mathrm{DA}}(n)$ and the contention window is initialized.

- (10i) and (10j) account for the special case when the back-off stage reaches $m$. Whether the transmission is successful or not, the contention window will be initialized to $W_{0}$.

We use $p_{i, j, k, l}$ to represent the steady-state probability (i, j, $\left.\mathrm{k}, \mathrm{l}\right)$. According to the non- null one-step transitional probabilities, we get

$$
p_{0, j, k, 0}=p_{0, j, k+1,0}+\frac{p_{0, j-1,0,0}\left[p_{\mathrm{RC}}(n)+\left(1-p_{\mathrm{RC}}(n)\right) p_{\mathrm{DA}}(n)\right]}{W_{j}} \quad 0 \leq k \leq W_{j}-2,1 \leq j \leq m .
$$


If $k=W_{j}-1$, we can get

$$
\begin{gathered}
p_{0, j, W_{j}-1,0}=\frac{p_{0, j-1,0,0}\left[p_{\mathrm{RC}}(n)+\left(1-p_{\mathrm{RC}}(n)\right) p_{\mathrm{DA}}(n)\right]}{W_{j}} \\
1 \leq j \leq m .
\end{gathered}
$$

For clarity, we assume

$$
\omega(n)=\left[p_{\mathrm{RC}}(n)+\left(1-p_{\mathrm{RC}}(n)\right) p_{\mathrm{DA}}(n)\right] .
$$

Then (12) can be rewritten as

$$
p_{0, j, W_{j}-1,0}=\frac{p_{0, j-1,0,0}}{W_{j}} \omega(n) \quad 1 \leq j \leq m .
$$

According to the relationship of (11) and (14), we have

$$
p_{0, j, k, 0}=p_{0, j-1,0,0} \frac{W_{j}-k}{W_{j}} \omega(n) \quad 1 \leq j \leq m, 0 \leq k \leq W_{j}-1 .
$$

Let $k=0$, (15) becomes

$$
p_{0, j, 0,0}=p_{0, j-1,0,0} \omega(n) \quad 1 \leq j \leq m .
$$

By substituting (16) into (15), we can derive

$$
p_{0, j, k, 0}=p_{0,0,0,0} \frac{W_{j}-k}{W_{j}} \omega(n)^{j} \quad 1 \leq j \leq m, 0 \leq k \leq W_{j}-1 .
$$

If we use $B(n)$ to represent the possibility that node $n$ is at back-off state, then we can derive

$$
\begin{aligned}
B(n) & =\sum_{j=0}^{m} \sum_{k=1}^{W_{j}-1} p_{0, j, k, 0}=\sum_{j=0}^{m} p_{0, j, 0,0} \sum_{k=1}^{W_{j}-1} \frac{W_{j}-k}{W_{j}}=\sum_{j=0}^{m} p_{0, j, 0,0} \frac{W_{j}-1}{2} \\
& =\frac{p_{0,0,0,0}}{2}\left[W_{0} \frac{1-(2 \omega(n))^{m+1}}{1-2 \omega(n)}-\frac{1-\omega(n)^{m+1}}{1-\omega(n)}\right] .
\end{aligned}
$$

When the back-off time counter reaches 0, the node will start to transmit the RTS frame. The possibility that node $n$ transmits RTS frame in a generic slot is

$$
\tau_{\mathrm{RTS}}(n)=\sum_{j=0}^{m} p_{0, j, 0,0}=p_{0,0,0,0} \frac{1-\omega(n)^{m+1}}{1-\omega(n)} .
$$

Only when node $n$ successfully transmits the RTS frame and receives the CTS frame, it can 
transmit the DATA frame. Thus, the possibility that node $n$ transmits DATA frame in a generic slot is

$$
\tau_{\mathrm{DATA}}(n)=\tau_{\mathrm{RTS}}(n)\left[1-p_{\mathrm{RC}}(n)\right]=p_{0,0,0,0} \frac{1-\omega(n)^{m+1}}{1-\omega(n)}\left[1-p_{\mathrm{RC}}(n)\right] .
$$

The saturation throughput of node $n$ is defined as the total number of bits sent per unit time, denoted by $S(n)$. If assuming $E[P]$ to be the average packet size, $S(n)$ can be expressed as:

$$
S(n)=\frac{\tau_{\mathrm{DATA}}(n)\left(1-p_{\mathrm{DA}}(n)\right) E[P]}{\sigma}
$$

\subsection{Transmission Process}

In the RTS-CTS transmission process, node $n$ will start a timer to wait for the CTS frame after transmitting the RTS frame. The duration of the timer should be long enough for transmitting a CTS frame. We assume that this time is equal to $L_{\text {CTS. }}$ Then, we can get that whether the RTS-CTS transmission is successful or failed, node $n$ will experience the same time slots, i.e., $\left(L_{\mathrm{RTS}}+L_{\mathrm{CTS}}\right)$. Similarly, node $n$ will experience $\left(L_{\mathrm{DATA}}+L_{\mathrm{ACK}}\right)$ time slots in the DATA-ACK transmission process.

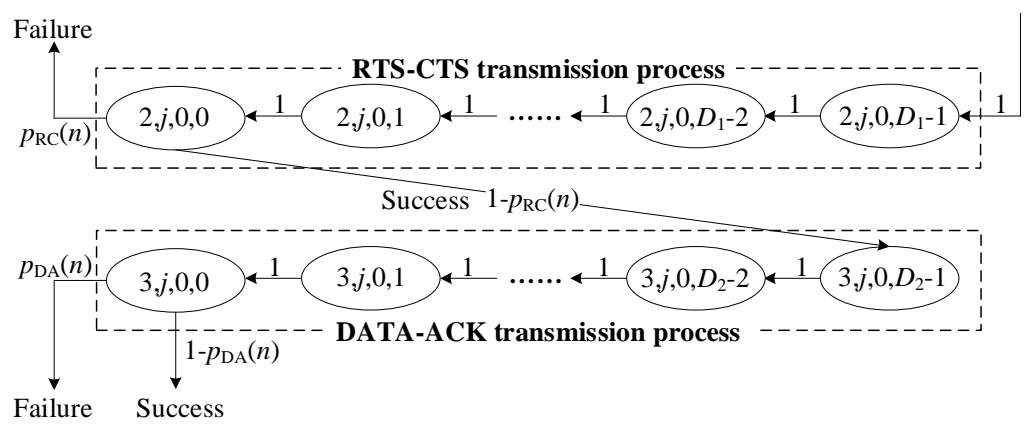

Fig. 6. Transmission process

Let $D_{1}=L_{\mathrm{RTS}}+L_{\mathrm{CTS}}, D_{2}=L_{\mathrm{DATA}}+L_{\mathrm{ACK}}$, then the transmission process can be depicted with Fig. 6. According to Fig. 6, the non-null one-step transition probabilities of transmission process are

$$
\left\{\begin{array}{ll}
p\left(2, j, 0, D_{1}-1 \mid 0, j, 0,0\right)=1 & 0 \leq j \leq m \\
p\left(3, j, 0, D_{2}-1 \mid 2, j, 0,0\right)=1-p_{\mathrm{RC}}(n) & 0 \leq j \leq m \\
p(2, j, 0, l-1 \mid 2, j, 0, l)=1 & 0 \leq j \leq m, 1 \leq l \leq D_{1}-1 \\
p(3, j, 0, l-1 \mid 3, j, 0, l)=1 & 0 \leq j \leq m, 1 \leq l \leq D_{2}-1
\end{array} .\right.
$$

The first equation in (21) illustrates the fact that node $n$ transmits the RTS frame with probability 1 when the back-off time counting number decreases to zero. The second equation expresses the fact that node $n$ will enter DATA-ACK transmission process with probability 1$p_{\mathrm{RC}}(n)$. The third and fourth equations represents that $l$ is decremented by 1 every time a slot passes during the RTS-CTS or DATA-ACK transmission process. 
Owing to the relationship of transition probabilities among between different states, we get

$$
\left\{\begin{array}{ll}
p_{2, j, 0, l}=p_{0, j, 0,0} & 0 \leq l \leq D_{1}-1 \\
p_{3, j, 0, l}=p_{0, j, 0,0}\left(1-p_{\mathrm{RC}}(n)\right) & 0 \leq l \leq D_{2}-1
\end{array} .\right.
$$

By means of (17) and (22), we can derive the probability that node $n$ is at the transmission state as

$$
\begin{aligned}
\tau^{\prime}(n) & =\sum_{j=0}^{m} \sum_{l=0}^{D_{1}-1} p_{2, j, 0, l}+\sum_{j=0}^{m} \sum_{l=0}^{D_{2}-1} p_{3, j, 0, l}=\left[D_{1}+D_{2}\left(1-p_{\mathrm{RC}}(n)\right)\right] \sum_{j=0}^{m} p_{0, j, 0,0} \\
& =p_{0,0,0,0}\left[D_{1}+D_{2}\left(1-p_{\mathrm{RC}}(n)\right)\right] \frac{1-\omega(n)^{m+1}}{1-\omega(n)}
\end{aligned}
$$

\subsection{Freezing Process}

When in back-off process, if node $n$ senses that the channel is busy, it will suspend the backoff time counter and enter the freezing process, as shown in Fig. 7. Assume that the duration expectation of the freezing process for node $n$ is $M(n)$, then the non- null transition probabilities can be written as

$$
\begin{cases}p(1, j, k, M(n)-1 \mid 0, j, k, 0)=p_{f}(n) & 0 \leq j \leq m, 1 \leq k \leq W_{j}-1 \\ p(1, j, k, l-1 \mid 1, j, k, l)=1 & 0 \leq j \leq m, 1 \leq k \leq W_{j}-1,1 \leq l \leq M(n)-1 . \\ p(0, j, k, 0 \mid 1, j, k, 0)=1 & 0 \leq j \leq m, 1 \leq k \leq W_{j}-1\end{cases}
$$

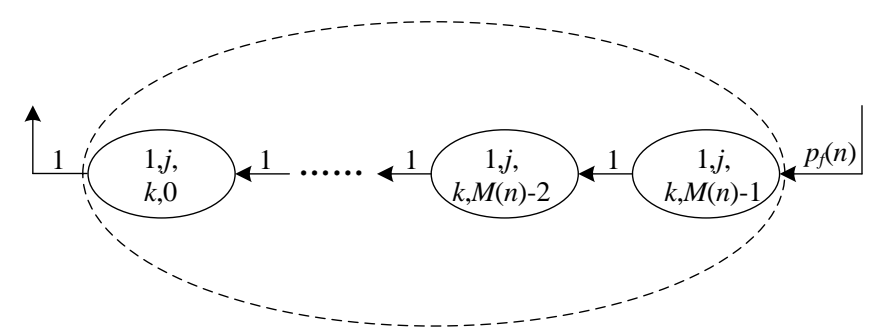

Fig. 7. Freezing process

The first equation in (25) accounts for the fact that the channel will become busy with probability $p_{f}(n)$. The second equation accounts for the fact that $l$ is reduced by 1 every time a slot passes during the freezing process. The third equation explains the fact that node $n$ continues the back-off process after the channel becomes idle. Note that

$$
\left\{\begin{array}{ll}
p_{1, j, k, M(n)-1}=p_{0,0,0,0} \frac{W_{j}-k}{W_{j}} \omega(n)^{j} p_{f}(n) & 0 \leq j \leq m, 1 \leq k \leq W_{j}-1, \\
p_{1, j, k, l-1}=p_{1, j, k, l} & 0 \leq j \leq m, 1 \leq k \leq W_{j}-1,0 \leq l \leq M(n)-1
\end{array} .\right.
$$

Based on the above formulations, all the values of $p_{i, j, k, l}$ can be expressed as the function of $p_{0,0,0,0}, p_{\mathrm{RC}}(n), p_{\mathrm{DA}}(n), p_{f}(n)$. By means of (18), (23), (25) and normalization condition, we 
finally get

$$
\begin{aligned}
1 & =\sum_{j=0}^{m} \sum_{k=0}^{W_{j}-1} p_{0, j, k, 0}+\sum_{j=0}^{m} \sum_{l=0}^{D_{1}-1} p_{2, j, 0, l}+\sum_{j=0}^{m} \sum_{l=0}^{D_{2}-1} p_{3, j, 0, l}+\sum_{j=0}^{m} \sum_{k=1}^{W_{j}-1} \sum_{l=0}^{M(n)-1} p_{1, j, k, l} \\
= & p_{0,0,0,0}\left[\frac{W_{0}\left(1+p_{f}(n) M(n)\right)}{2}\right] \frac{1-(2 \omega(n))^{m+1}}{1-2 \omega(n)} \\
& +p_{0,0,0,0}\left[\frac{\left(1-p_{f}(n) M(n)\right)}{2}+D_{1}+D_{2}\left(1-p_{\mathrm{RC}}(n)\right)\right] \frac{1-\omega(n)^{m+1}}{1-\omega(n)} .
\end{aligned}
$$

For convenience, we assume that

$$
\left\{\begin{array}{l}
p_{1}=\frac{\left(1+p_{f}(n) M(n)\right)}{2} \\
p_{2}=\frac{\left(1-p_{f}(n) M(n)\right)}{2}+D_{1}+D_{2}\left(1-p_{\mathrm{RC}}(n)\right)
\end{array}\right.
$$

By combining (26) and (27), $p_{0,0,0,0}$ can be expressed as

$$
p_{0,0,0,0}=\frac{1}{p_{1} W_{0} \frac{1-(2 \omega(n))^{m+1}}{1-2 \omega(n)}+p_{2} \frac{1-\omega(n)^{m+1}}{1-\omega(n)}} .
$$

Note that all variables except $p_{f}(n)$ and $M(n)$ are related to $p_{0,0,0,0}$. In other words, if $p_{f}(n)$ and $M(n)$ are determined, $p_{0,0,0,0}$ will be determined, too. Inspired by [30], we use a continuous Markov chain with an iterative way to compute $p_{f}(n)$ and $M(n)$.

In the continuous Markov chain model for directional CSMA/CA based MAC protocols, we suppose that the data packet arrival rate of the node $n$ follows a Poisson distribution with mean $\lambda(n)$ and the average data packet length is $1 / \rho(n)$. If we use $J$ to represents the independent set of nodes which can transmit simultaneously because of space division multiplexing property of directional antennas, the steady-state probability of $J$ can be expressed as

$$
Q(J)=\left(\prod_{n \in J} \frac{\lambda(n)}{\rho(n)}\right) Q(\phi),
$$

where $Q(\phi)$ is the probability that no node stays in transmission process. With the normalization condition of $Q(J)$, we can derive

$$
Q(\phi)=\left[\sum_{\text {all } J} \prod_{n \in J} \frac{g(n)}{u(n)}\right]^{-1},
$$

In the fixed-length slot Markov chain model, the probability that a node continues the backoff process after a time slot $\sigma$ is 


$$
p_{i}(n)=\left[1-\tau_{\mathrm{RTS}}(n)\right]\left[1-p_{f}(n)\right] \text {. }
$$

In the continuous Markov chain model, it is equivalent to the probability that no transmission occurs from the channel is idle during the interval $\sigma$. Let $\Lambda(n)$ be the aggregate scheduling rate of node $n$ and its neighbors from the state that node $n$ can transmit, it can be expressed as

$$
p_{i}(n)=e^{-\Lambda(n) \sigma}
$$

By relations of (32) and (33), we can derive

$$
p_{f}(n)=1-\frac{e^{-\Lambda(n) \sigma}}{1-\tau_{\mathrm{RTS}}(n)}
$$

The calculation of $\Lambda(n)$ is as follows:

$$
\Lambda(n)=\lambda(n)+\sum_{n^{\prime} \in T(n)} B\left(n^{\prime} \mid n\right) \lambda\left(n^{\prime}\right)
$$

where $T(\mathrm{n})$ represents the set of neighbors of node $n, B\left(n^{\prime} \mid n\right)$ represents the probability that node $n^{\prime}$ is allowed to transmit under the condition that node $n$ can transmit, it is

$$
B\left(n^{\prime} \mid n\right)=\frac{B\left(n^{\prime}, n\right)}{B(n)}=\frac{\sum_{J \subset \overline{T(n) \cup T\left(n^{\prime}\right)}}\left(\prod_{n \in J} \frac{\lambda(n)}{\rho(n)}\right)}{\sum_{J \subset \bar{T}(n)}\left(\prod_{n \in J} \frac{\lambda(n)}{\rho(n)}\right)} .
$$

$B(n)$ is the probability that node $n$ senses the communication channel is idle, which is equal to the sum of the steady-state probability of all the independent set that can transmit simultaneously. Thus, we get

$$
B(n)=\sum_{J \subset \bar{T}(n)} Q(H)=\frac{\sum_{J \subset \bar{T}(n)}\left(\prod_{n \in J} \lambda(n) / \rho(n)\right)}{\sum_{\text {all } J}\left(\prod_{n \in J} \lambda(n) / \rho(n)\right)} .
$$

Based on (18), (33) and (37), we have established a relationship between the fixed-length Markov chain model and the continuous Markov chain model. By substituting the initial value of $\lambda(n)$ and $\rho(n)$ into (37), we can calculate the value of $B(n)$. Then, by relations (19), (34), (35) and (36), $p_{f}(n)$ can be expressed as the function of $p_{0,0,0,0} . M(n)$ is further derived by substituting $p_{f}(n)$ into (18). With the derivation of $p_{f}(\mathrm{n})$ and $M(n)$, all the variables of the fixed-length Markov chain model can be calculated according to (27). Substituting their values into (21), the per stream saturation throughput is finally obtained.

By using the continuous Markov chain model, the saturation per stream saturation throughput can be expressed as: 


$$
s(n)=B(n) \lambda(n)[1-\omega(n)]
$$

We use (38) to update the value of $\lambda(n)$, and the other variables are further updated accordingly. These variables are in turn used to update the value of the per-flow saturation throughput. The above steps are repeated until the result converges. The convergence result of the per-flow saturation throughput is exactly what we need.

\section{Model Validation}

In this section, we adopt the network simulator NS3 to validate our model by comparing the numerical results with the simulation results. Consider a topology with 30 flows randomly distributed in a $250 \mathrm{~m} \times 250 \mathrm{~m}$ area, as shown in Fig. 8. The sender of flow $n$ is denoted as node $2 n-1$ which are filled with grey, and the receiver of flow $n$ is denoted as node $2 n$ which are filled with black. Assume that all nodes are at saturated condition, i.e., all of them always have at least one packet to send after a successful transmission. We set the packet size of each flow to a fixed value of 256 bytes. Other parameters used to obtain numerical and simulation results are listed in Table 3.

Table 3. Parameters Used to Obtain Numerical and Simulation Results

\begin{tabular}{|c|c|c|c|}
\hline Parameters & Value & Parameters & Value \\
\hline \hline Simulation time & $100 \mathrm{~s}$ & SNR threshold & $10 \mathrm{~dB}$ \\
\hline Channel bandwidth & $2 \mathrm{Mbps}$ & Retransmission limit & 4 \\
\hline Slot time & $20 \mu \mathrm{s}$ & SIFS & $10 \mu \mathrm{s}$ \\
\hline DIFS & $50 \mu \mathrm{s}$ & Propagation model & Two-ray \\
\hline PHY header & $192 \mathrm{bits}$ & MAC header & $224 \mathrm{bits}$ \\
\hline
\end{tabular}

\subsection{Transmission Probability}

Fig. 9 gives the comparison results of RTS and DATA transmission probability with different beamwidth. Overall, we observe that the analysis result is very close to the simulation result in directional CSMA/CA based Ad hoc networks. On the one hand, the RTS transmission probability is higher than the DATA transmission probability for most flows when the beamwidth is the same. That is because if the sender does not receive the CTS frame after transmitting the RTS frame, it will not transmit the DATA frame. On the other hand, the narrower beamwidth allows more spatial reuse, and the more idle time of the channel sensed by a few flows. As a result, this fraction of flows has more opportunities to access the channel. That is, the narrower the beam is, the higher the RTS and DATA transmission probabilities are for a few flows. 


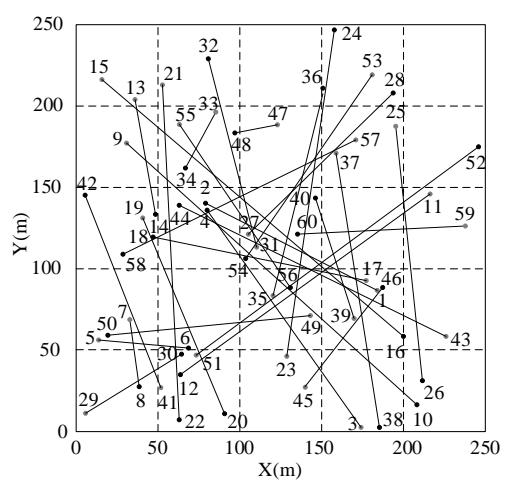

Fig. 8. A random topology with 30 flows

As mentioned above, the network environment faced by each flow is different considering the deafness and directional hidden terminal problems in a directional CSMA/CA based Ad hoc networks. This disparity of flows leads to the different RTS and DATA transmission probability of each flow. For example, in Fig. 9(a), when the beamwidth is 90 degrees, the RTS transmitting probability of stream 15 is higher than other flows. As can be seen from Fig. 8, node 29 and node 30 are the corresponding sender and receiver of flow 15 , respectively. Note that there are no other nodes except node 29 in the beam direction of node 30, the RTS frame is always received successfully. Because of fewer collisions of transmission, the contention window size of node 29 will remain near the minimum, so it is more likely to choose a smaller back-off value. Therefore, the RTS transmitting probability of stream 15 is higher than other flows.

In contrast, flow 21 has a quite low RTS transmission probability because there are other five transmitting nodes that are aligned with node 42: node 3, 17, 31, 39 and 43 . When the sender of flow 21, i.e. node 41, starts to transmit the RTS frame, if one of these nodes finishes the back-off process and starts transmission during the RTS transmission process, collisions will occur at node 42 . It will result in doubling the contention window size of node 41 and retransmitting the RTS frame. If the retransmission is unsuccessful, the contention window will become twice than before again. When it is equal to the retransmission limit, the packet will be discarded. Hence, the RTS transmission probability of this flow is lower than other flows. Similarly, when the beamwidth turns to be 60 degrees or 30 degrees, the disparity in RTS transmission probability still exists. Further observations show that when a flow has low RTS transmission probability, the DATA transmission probability will be lower in most cases. If the RTS transmission probability of one flow is high, the DATA transmission probability is substantially the same or slightly decreased.

In omni-directional CSMA/CA based Ad hoc networks, as shown in Fig. 9, the RTS and DATA transmission probability of flows are almost the same because of the equal access to the channel. Compared with directional CSMA/CA based Ad hoc networks, we can see that the RTS and DATA transmission probability of omni-directional flows are higher than most of the directional flows when the beamwidth is 90 degrees and 60 degrees. However, under the condition that the beamwidth is 30 degrees, there are more than one-third of directional flows which have higher RTS and DATA transmission probability than omni-directional flows. The reason for this increment is that when the antenna is narrow beam antenna, the collision zone of flows is reduced, so the amount of potential conflicting streams is reduced accordingly. That is to say, the transmission of flows is less interfered by their neighbor flows. As a result, the RTS and DATA transmission probability of flows have a significant increase with narrower beamwidth. 


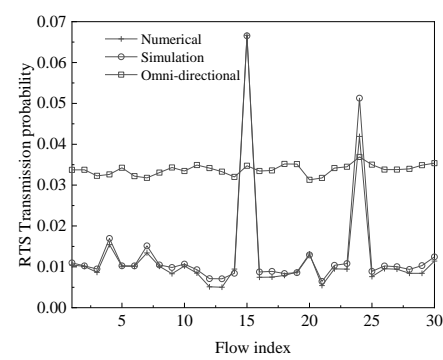

(a) RTS transmission probability (90 degrees)

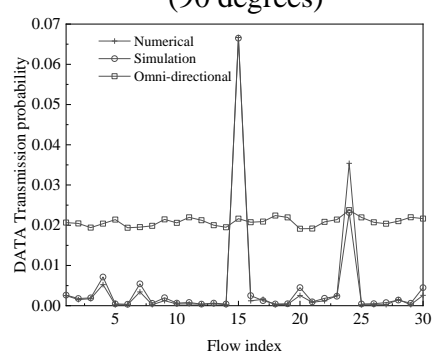

(b)DATA transmission probability (90 degrees)

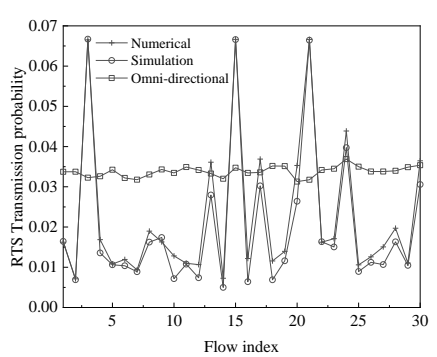

(c) RTS transmission probability (60 degrees)

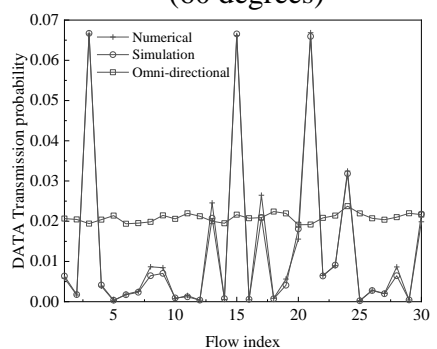

(d) DATA transmission probability (60 degrees)

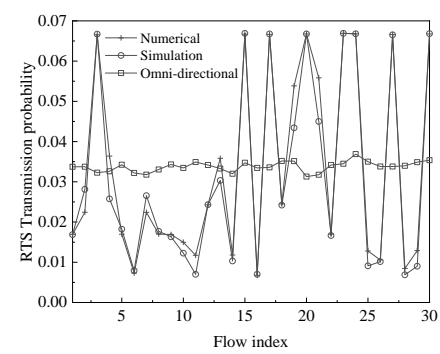

(e) RTS transmission probability (30 degrees)

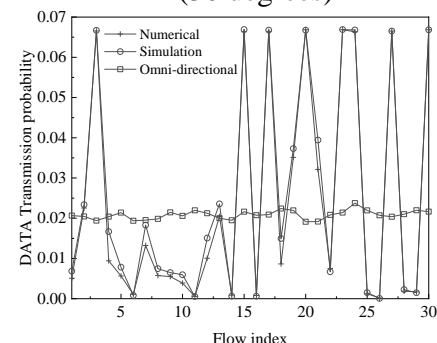

(f) DATA transmission probability (30 degrees)

Fig. 9. Per flow transmission probability

\subsection{Collision Probability}

Fig. 10 shows the RTS and DATA collision probability of flows in the directional CSMA/CA based Ad hoc networks with the random topology. Intuitively, using directional antennas will reduce the number of nodes that are in competition of the tagged flow, so the collision probability of directional flows should be lower than that of omni-directional flows. However, when the beamwidth is 90 degrees and 60 degrees, as shown in Fig. 10(a), (b), (c), and (d), the RTS and DATA collision probability of most directional flows are higher than that of omni-directional flows. Although the directional antennas can increase the space division multiplexing, the deafness and directional hidden terminal problems greatly weaken the gain of directional antennas, resulting in higher actual RTS and DATA collision probability than omni-directional networks. When the beamwidth is further narrowed to 30 degrees, the advantage of directional antennas becomes noticeable. There are more than half of directional flows whose RTS collision probability is lower than omni-directional flows.

In Fig. 10(a), (c), and (e), the RTS collision probability of stream 15 is almost zero. Since there are no other conflicting nodes in the collision area of node 30 , the packets transmitted by the sender of flow 15 can be received successfully. However, the collision probability of flows that have multiple conflicting nodes in the collision area is relatively high. For example, besides the sender of flow 12 , i.e. node 23 , the beam direction of node 24 is also aligned with nodes $27,29,35,45$ and 51 . Whereas node 27 has a high transmission probability as shown in Fig. 10, and it is in the persistent collision area of node 24 . Hence, the collision probability of flow 12 is high due to the existence of these conflicting nodes.

For a specified flow, its DATA collision probability is usually lower than the RTS collision probability. The DATA collision probability will not be counted unless the CTS frame is correctly received. In omni-directional CSMA/CA based Ad hoc networks, as shown in Fig. 10, the DATA collision probability is almost zero. That is because the RTS/CTS handshake mechanism can effectively reduce the collisions of DATA transmission process. But in directional CSMA/CA based Ad hoc networks, because of deafness and directional hidden 
terminal problems, the DATA collision probability may still exist even if the CTS frame is correctly received.

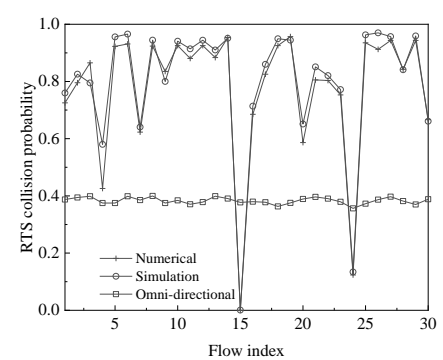

(a) RTS collision probability (90 degrees)

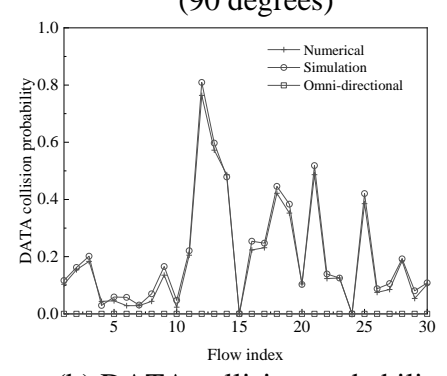

(b) DATA collision probability (90 degrees)

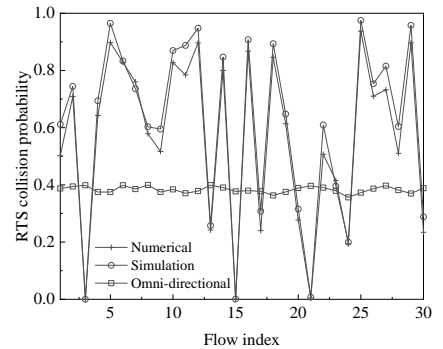

(c) RTS collision probability (60 degrees)

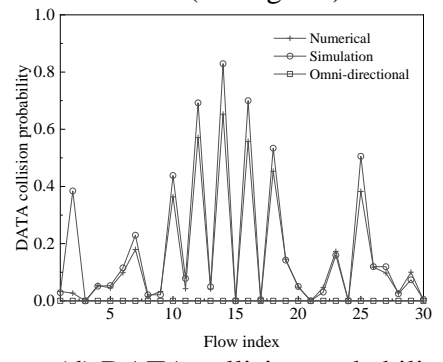

(d) DATA collision probability (60 degrees)

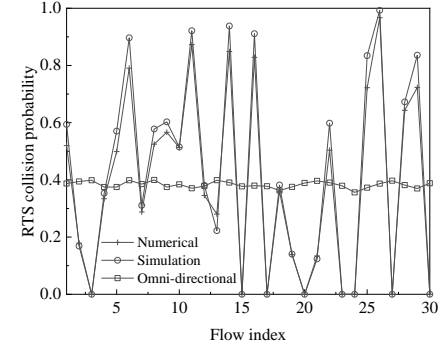

(e) RTS collision probability (30 degrees)

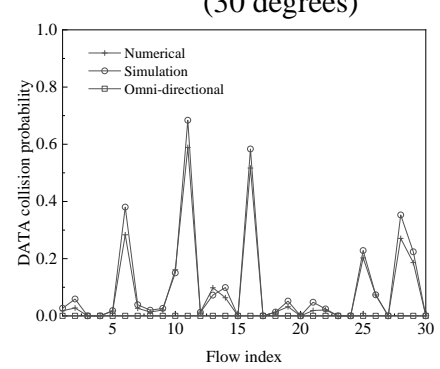

(f) DATA collision probability (30 degrees)

Fig. 10. Per flow collision probability

\subsection{Saturation Throughput}

Fig. 11(a), (c), and (e) illustrate the analytical and simulation results of the saturated throughput of per-flow. We can see that the analytical results match well with the simulation results from them, which verifies the accuracy of our analytical model. Further observation, we note that there is a big disparity in the saturation throughput among flows. Some flows can achieve very high saturation throughput while others have a very low saturation throughput, and even a few flows get starved. Although using directional antennas can increase the saturation throughput of the whole network by increasing the spatial reuse, it may lead to the unfairness problem among flows compared with omni-directional antennas.

Comparing Fig. 9, Fig. 10, and Fig. 11, we observe that the overall trends of the saturation throughput of per-flow is similar to that of the transmission probability and contrary to that of the collision probability. There are also some special cases, because the saturation throughout of per-flow is not only related to the transmission probability and collision probability, but also related to the suspension probability. For example, in Fig. 11(a), the saturation throughput of stream 15 is lower than flow 24. Review Fig. 9 and Fig. 10, we can see that the RTS and DATA transmission probabilities of stream 15 are both higher than stream 24, and the collision probability of both flows are almost zero. However, the corresponding sender of flow 15, i.e. node 29 , is also aligned with other 7 nodes except besides its receiver. If one or more of these interfere nodes start the transmission process when node 29 is in the back-off process, node 29 will suspend its back-off time counter and enter the freezing process. That is to say, the suspension probability of flow 15 is relatively high. On the other hand, the amount of nodes aligned with the sender of flow 24 (node 47) is only 4, so the suspension probability of node 47 is lower than node 29. Combining the effects of transmission probability, collision probability and suspension probability, the actual saturation throughput of stream 15 is lower 
than that of flow 24.

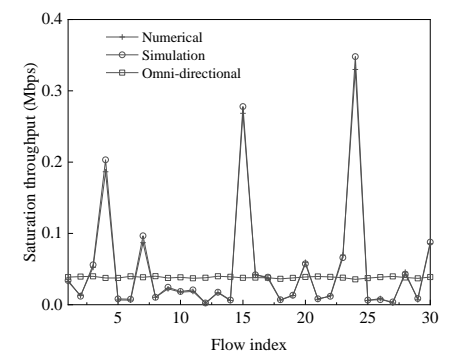

(a) Saturation throughput (90 degrees)

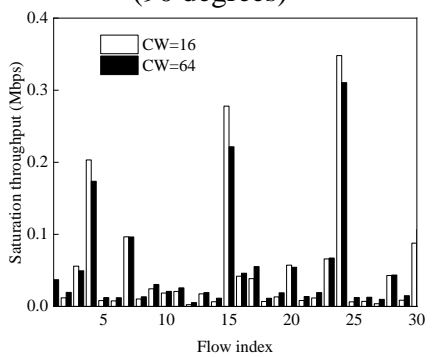

(b) Saturation throughput (90 degrees)

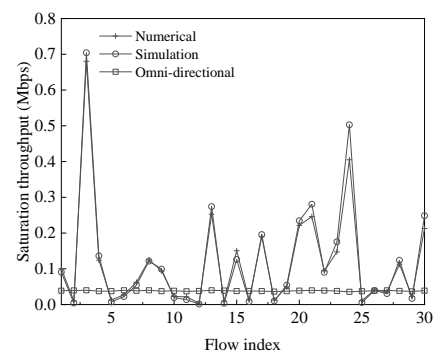

(c) Saturation throughput (60 degrees)

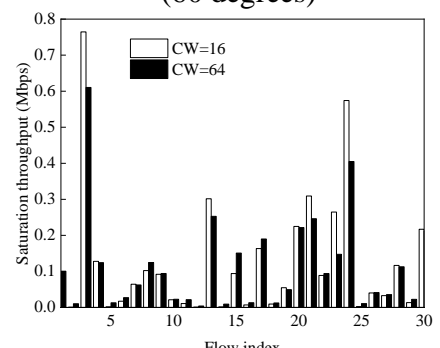

(d) Saturation throughput (60 degrees)

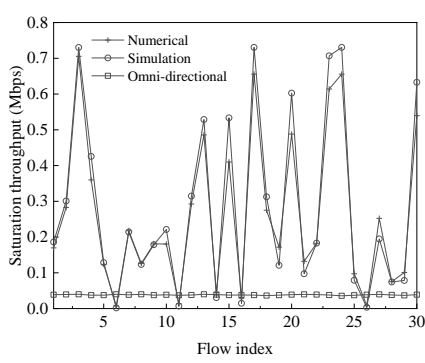

(e) Saturation throughput (30 degrees)

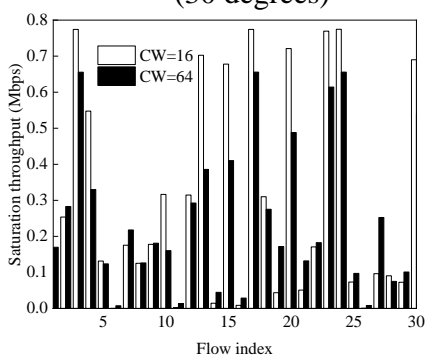

(f) Saturation throughput (30 degrees)

Fig. 11. Per flow saturation throughput

Fig. 11(b), (d), and (f) gives the per-flow saturation throughput with different minimum contention window size. The change of saturation throughput of different flows is different when the minimum contention window size becomes greater. Generally speaking, the saturation throughput of flows with high saturation throughput decreases while the saturation throughput of flows with low saturation throughput increases. That is because the flows with high saturation throughput have a high transmission probability and low collision probability. When the minimum contention window size increases, the transmission probability decreases significantly while the collision probability remains almost the same. Therefore, the saturation throughput of these flows decreases. For those flows with low saturation throughput, their collision probability is greatly decreased with the increase of the minimum contention window size, so their saturation throughput increases instead.

\section{Conclusion}

In this paper, we have presented a novel analytical model for analyzing the saturated throughput disparity of flows in directional CSMA/CA based Ad hoc networks. The analytical model fully considers both the effect of deafness and directional hidden terminal problems through a four-dimensional Markov chain which is based on the notion of the fixed-length slot. By analyzing the back-off process, transmission process and freezing process of the tagged node, we deduce the per stream saturation throughput. The accuracy of the proposed model is validated by extensive simulations. Our work provides a deeper understanding of the behavior of CSMA/CA based MAC protocols in directional Ad hoc networks and can promote the improvement of unfairness problem in the design of directional MAC protocols. 


\section{References}

[1] P. Sun and N. Samaan, "A Novel VANET-Assisted Traffic Control for Supporting Vehicular Cloud Computing," IEEE Transactions on Intelligent Transportation Systems, 2020. Article (CrossRef Link)

[2] I. García-Magariño, R. Lacuesta, M. Rajarajan, and J. Lloret, "Security in networks of unmanned aerial vehicles for surveillance with an agent-based approach inspired by the principles of blockchain," Ad Hoc Networks, vol. 86, pp. 72-82, 2019. Article (CrossRef Link)

[3] X. Hu, J. Cheng, M. Zhou, B. Hu, X. Jiang, Y. Guo, K. Bai, and F. Wang, "Emotion-aware cognitive system in multi-channel cognitive radio ad hoc networks," IEEE Communications Magazine, vol. 56, no. 4, pp. 180-187, 2018. Article (CrossRef Link)

[4] F. De Rango, G. Potrino, M. Tropea, A. F. Santamaria, and P. Fazio, "Scalable and ligthway bioinspired coordination protocol for FANET in precision agriculture applications," Computers \& Electrical Engineering, vol. 74, pp. 305-318, 2019. Article (CrossRef Link)

[5] M. Gerasimenko, D. Moltchanov, M. Gapeyenko, S. Andreev, and Y. Koucheryavy, "Capacity of multiconnectivity mmWave systems with dynamic blockage and directional antennas," IEEE Transactions on Vehicular Technology, vol. 68, no. 4, pp. 3534-3549, 2019. Article (CrossRef Link)

[6] J. Guo, P. Walk, and H. Jafarkhani, "Optimal deployments of UAVs with directional antennas for a power-efficient coverage," IEEE Transactions on Communications, vol. 68, no. 8, pp. 5159-5174, 2020. Article (CrossRef Link)

[7] P. Chu, J. A. Zhang, X. Wang, Z. Fei, G. Fang, and D. Wang, "Interference Characterization and Power Optimization for Automotive Radar With Directional Antenna," IEEE Transactions on Vehicular Technology, vol. 69, no. 4, pp. 3703-3716, 2020. Article (CrossRef Link)

[8] R. R. Choudhury, X. Yang, R. Ramanathan, and N. H. Vaidya, "Using directional antennas for medium access control in ad hoc networks," in Proc. of the $8^{\text {th }}$ Annual International Conference on Mobile Computing and Networking, pp. 59-70, 2002. Article (CrossRef Link)

[9] E. Shihab, L. Cai, and J. Pan, "A distributed asynchronous directional-to-directional MAC protocol for wireless ad hoc networks," IEEE Transactions on Vehicular Technology, vol. 58, no. 9, pp. 5124-5134, 2009. Article (CrossRef Link)

[10] D. N. M. Dang, V. Nguyen, H. T. Le, C. S. Hong, and J. Choe, "An efficient multi-channel MAC protocol for wireless ad hoc networks," Ad Hoc Networks, vol. 44, pp. 46-57, 2016. Article (CrossRef Link)

[11] X. Li, F. Hu, J. Qi, and S. Kumar, "Systematic Medium Access Control in Hierarchical Airborne Networks with Multi-Beam and Single-Beam Antennas," IEEE Transactions on Aerospace and Electronic Systems, vol. 55, no. 2, pp. 706-717, 2018. Article (CrossRef Link)

[12] Z. Zheng, A. K. Sangaiah, and T. Wang, "Adaptive communication protocols in flying ad hoc network,” IEEE Communications Magazine, vol. 56, no. 1, pp. 136-142, 2018. Article (CrossRef Link)

[13] J. Yang, G. Qiao, Q. Hu, J. Zhang, and G. Du, “A Dual Channel Medium Access Control (MAC) Protocol for Underwater Acoustic Sensor Networks Based on Directional Antenna," Symmetry, vol. 12, no. 6, 2020. Article (CrossRef Link)

[14] A. Akhtar and S. C. Ergen, "Directional MAC protocol for IEEE 802.11 ad based wireless local area networks," Ad Hoc Networks, vol. 69, pp. 49-64, 2018. Article (CrossRef Link)

[15] J. M. Me Biomo, M. Kunz, and M. St-Hilaire, "Directional antennas in FANETs: A performance analysis of routing protocols," in Proc. of International Conference on Selected Topics in Mobile and Wireless Networking (MoWNeT), pp. 1-8, 2017. Article (CrossRef Link)

[16] O. Bazan and M. Jaseemuddin, "A Survey On MAC Protocols for Wireless Adhoc Networks with Beamforming Antennas," IEEE Communications Surveys \& Tutorials, vol. 14, no. 2, pp. 216-239, 2012. Article (CrossRef Link)

[17] Y. Qu, B. Ng, and M. Homer, "Optimising channel assignment to prevent flow starvation and improve fairness for planning single radio WMNs in built environments," Computer networks, vol. 129, pp. 215-231, 2017. Article (CrossRef Link) 
[18] S. Temel and I. Bekmezci, "LODMAC: Location oriented directional MAC protocol for fanets," Computer Networks, vol. 83, pp. 76-84, 2015. Article (CrossRef Link)

[19] G. Bianchi, "Performance analysis of the IEEE 802.11 distributed coordination function," IEEE Journal on Selected Areas in Communications, vol. 18, no. 3, pp. 535-547, 2000. Article (CrossRef Link)

[20] E. Felemban and E. Ekici, "Single hop IEEE 802.11 DCF analysis revisited: Accurate modeling of channel access delay and throughput for saturated and unsaturated traffic cases," IEEE Transactions on Wireless Communications, vol. 10, no. 10, pp. 3256-3266, 2011. Article (CrossRef Link)

[21] L. Dai and X. Sun, "A unified analysis of IEEE 802.11 DCF networks: Stability, throughput, and delay,” IEEE Transactions on Mobile Computing, vol. 12, no. 8, pp. 1558-1572, 2012. Article (CrossRef Link)

[22] F. Babich and M. Comisso, "Throughput and delay analysis of 802.11-based wireless networks using smart and directional antennas," IEEE Transactions on Communications, vol. 57, no. 5, pp. 1413-1423, 2009. Article (CrossRef Link)

[23] O. Bazan and M. Jaseemuddin, "Performance analysis of directional CSMA/CA in the presence of deafness," IET communications, vol. 4, no. 18, pp. 2252-2261, 2010. Article (CrossRef Link)

[24] Y. Chen, J. Liu, X. Jiang, and O. Takahashi, “Throughput analysis in mobile ad hoc networks with directional antennas," Ad Hoc Networks, vol. 11, no. 3, pp. 1122-1135, 2013. Article (CrossRef Link)

[25] M. Kiran and P. Rajalakshmi, "Saturated throughput analysis of IEEE 802.11 ad EDCA for high data rate 5G-IoT applications," IEEE Transactions on Vehicular Technology, vol. 68, no. 5, pp. 4774-4785, 2019. Article (CrossRef Link)

[26] W. Wu, N. Cheng, N. Zhang, P. Yang, K. Aldubaikhy, and X. Shen, "Performance Analysis and Enhancement of Beamforming Training in 802.11 ad," IEEE Transactions on Vehicular Technology, vol. 69, no. 5, pp. 5293-5306, 2020. Article (CrossRef Link)

[27] A. Tsertou and D. I. Laurenson, "Revisiting the hidden terminal problem in a CSMA/CA wireless network," IEEE Transactions on Mobile Computing, vol. 7, no. 7, pp. 817-831, 2008. Article (CrossRef Link)

[28] K. Xu, M. Gerla, and S. Bae, "How effective is the IEEE 802.11 RTS/CTS handshake in ad hoc networks," in Proc. of Global Telecommunications Conference, pp. 72-76, 2002. Article (CrossRef Link)

[29] J. S. Reigadas, A. Martinez-Fernandez, J. Ramos-Lopez, and J. Seoane-Pascual, "Modeling and Optimizing IEEE 802.11 DCF for Long-Distance Links," IEEE Transactions on Mobile Computing, vol. 9, no. 6, pp. 881-896, 2010. Article (CrossRef Link)

[30]M. Garetto, T. Salonidis, and E. W. Knightly, "Modeling Per-Flow Throughput and Capturing Starvation in CSMA Multi-Hop Wireless Networks," IEEE/ACM Transactions on Networking, vol. 16, no. 4, pp. 864-877, 2008. Article (CrossRef Link) 


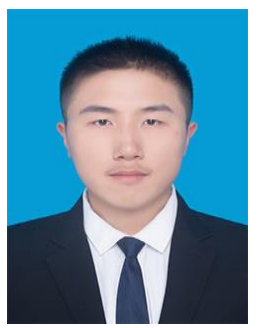

Jianrui Fan received the bachelor's degree in information engineering from the Nanjing University of Aeronautics and Astronautics in 2018, where he is currently pursuing the master's degree with the Communication and Information System. His research interest includes the design and optimization of the MAC protocol for wireless ad hoc networks.

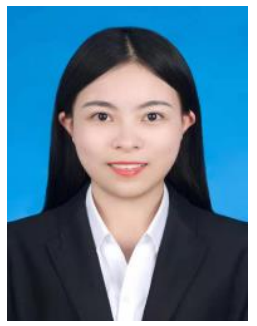

Xinru Zhao received the M.Sc. degree in communication engineering from the Nanjing University of Aeronautics and Astronautics, China, in 2018. Her research interest includes the design and performance analysis of the MAC protocol for wireless ad hoc networks.

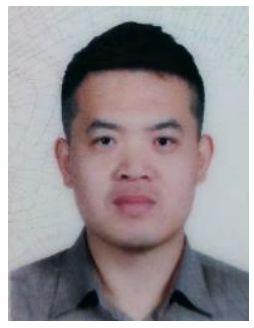

Wencan Wang is master student, and his interest is telemetry and remote control. He has been engaged in aircraft measurement and control for a long time and is familiar with the demonstration and application of telemetry, radar, optics and other measurement equipment.

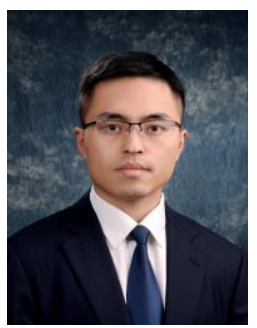

Shengsuo Cai received the M.Sc. degree in communication engineering from the Nanjing University of Aeronautics and Astronautics, China, in 2014, where he is currently with the College of Electronic and Information Engineering. His research interest includes the design and performance analysis of the MAC protocol for wireless ad hoc networks.

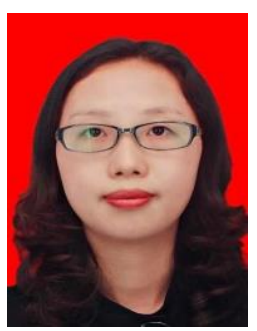

Lijuan Zhang received the B.Eng. degree in information security from the University of Southwest Jiaotong University, Chengdu, China, in 2010, and the Ph.D. degree in electronic engineering from James Cook University, Cairns, Australia, in 2018. She is currently an Associate Professor with the College of Electronic and Information Engineering, Nanjing University of Aeronautics and Astronautics, Nanjing, China. Her research interests include RFID communication and MAC protocol design. 